\title{
The Viability of Organic Dyes in Luminescent Down-Shifting Layers for the Enhancement of Si Solar Cell Efficiency
}

\author{
Aaron Glenn \\ Trinity College Dublin, Ireland, glenna@tcd.ie \\ Conor McLoughlin \\ Technological University Dublin, cmclouc10@tcd.ie \\ Hind Ahmed \\ Trinity College Dublin, Ireland, hahmed@tcd.ie
}

See next page for additional authors

Follow this and additional works at: https://arrow.tudublin.ie/engschcivart

Part of the Civil Engineering Commons, and the Environmental Engineering Commons

\section{Recommended Citation}

Glenn, A., Loughlin, C. M., Hind, A., Akbari, H., Chandra, S., \& McCormack, S. (2020). The viability of organic dyes in luminescent down-shifting layers for the enhancement of si solar cell efficiency. Materials Science Forum, 995, 71-76. doi:http://dx.doi.org/10.4028/www.scientific.net/MSF.995.71

This Article is brought to you for free and open access by the School of Civil and Structural Engineering at ARROW@TU Dublin. It has been accepted for inclusion in Articles by an authorized administrator of ARROW@TU Dublin. For more information, please contact arrow.admin@tudublin.ie, aisling.coyne@tudublin.ie, gerard.connolly@tudublin.ie. Funder: European Research Council; Science Foundation Ireland

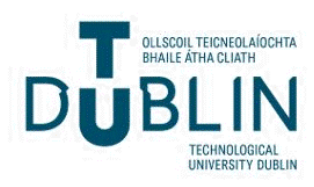




\section{Authors}

Aaron Glenn, Conor McLoughlin, Hind Ahmed, Hoda Akbari, Subhash Chandra, and Sarah McCormack 


\title{
The Viability of Organic Dyes in Luminescent Down-Shifting Layers for the Enhancement of Si Solar Cell Efficiency
}

\author{
A. Glenn ${ }^{1,2, a}$, C. McLoughlin ${ }^{1,2, b}$, H. Ahmed ${ }^{1}$, H. Akbari ${ }^{1}$, S. Chandra ${ }^{1}$ \\ and S. McCormack ${ }^{1}$ \\ ${ }^{1}$ School of Engineering, Trinity College Dublin, Dublin, Ireland \\ ${ }^{2}$ Technological University Dublin, Dublin, Ireland \\ aglenna@tcd.ie, ${ }^{b}$ mclouc10@tcd.ie
}

\begin{abstract}
Keywords: Luminescent Down-Shifting Layer, Lumogen Dye, Silicon Solar Cell, External Quantum Efficiency, Spin-coating.
\end{abstract}

\begin{abstract}
The main energy losses in solar cells are related to spectral losses where high energy photons are not used efficiently, and energy is lost via thermalization which reduces the solar cell's overall efficiency. A way to tackle this is to introduce a luminescent down-shifting layer (LDS) to convert these high energy photons into a lower energy bracket helping the solar cell to absorb them and thus generating a greater power output. In this paper, lumogen dye Violet 570 has been used as LDS coated films of $10 \mu \mathrm{m}$ and $60 \mu \mathrm{m}$ placed on top of Si solar cells. The dye was incorporated into polymer films of Polyvinyl Butyral (PVB) and Polymethyl Methacrylate (PMMA) after which they were tested for their absorption, transmission and emission properties. Once optimised layers had been determined, they were deposited directly onto silicon solar cells and the external quantum efficiency (EQE) of the Si solar cells were measured with and without the LDS layers. The resulting graphs have shown an increase of up to $2.9 \%$ in the overall EQE efficiency after the lumogen films had been applied.
\end{abstract}

\section{Introduction}

It is clear that a major problem in todays' society is the way that human beings are producing power. They are heavily reliant on fossil fuels which are a finite resource and one that is depleting rapidly. The problem with fossil fuels is not only that they are becoming increasingly more expensive but that they are also very damaging to the environment. There is already a strain on our energy production levels and yet the population is expected to increase from 7.2 billion (2015) to 9.7 billion by 2050 , meaning that the current system is just not sustainable [1]. Solar energy supply is expected to increase from $32 \%$ in 2030 to $73 \%$ by 2050 becoming the world's main energy source. This will greatly help reduce Greenhouse Gas Emissions from 30,000 $\mathrm{MtCO}_{2 \text { eq }}(2015)$ to zero in 2050 across all energy sectors if targets are met [1]. The future goal of the current work is to improve the efficiency and reduce the cost of renewable energy systems in order to incentivize the swap over to renewable energy sources.

This project involves the optimization of luminescent materials for photovoltaic applications. It aims at working with different types of PV solar cell and attempts to first produce a polymer layer to be placed on top of the solar cell with high transmission properties and then to add a lumogen dye to this layer to introduce the LDS effect.

\section{Theory}

Photovoltaic solar cells suffer from a low external quantum efficiency (EQE) in the ultra-violet to blue range of the electromagnetic spectrum. A purely optical approach to increase the short- $\lambda$ response of the cell is the use of LDS. In LDS, a luminescent material is encapsulated in a polymer sheet on top of a solar cell, it captures high energy photons and re-emits them at longer wavelengths that better match the photosensitivity spectral response of the cell [2,3]. As a result, more electron hole pairs are created, this means that more current is generated in the cell and this in turn gives a 
higher EQE $[4,5]$. The optical process involves a planar luminescent sheet deposited on top of the PV cell as illustrated in Fig. 1.

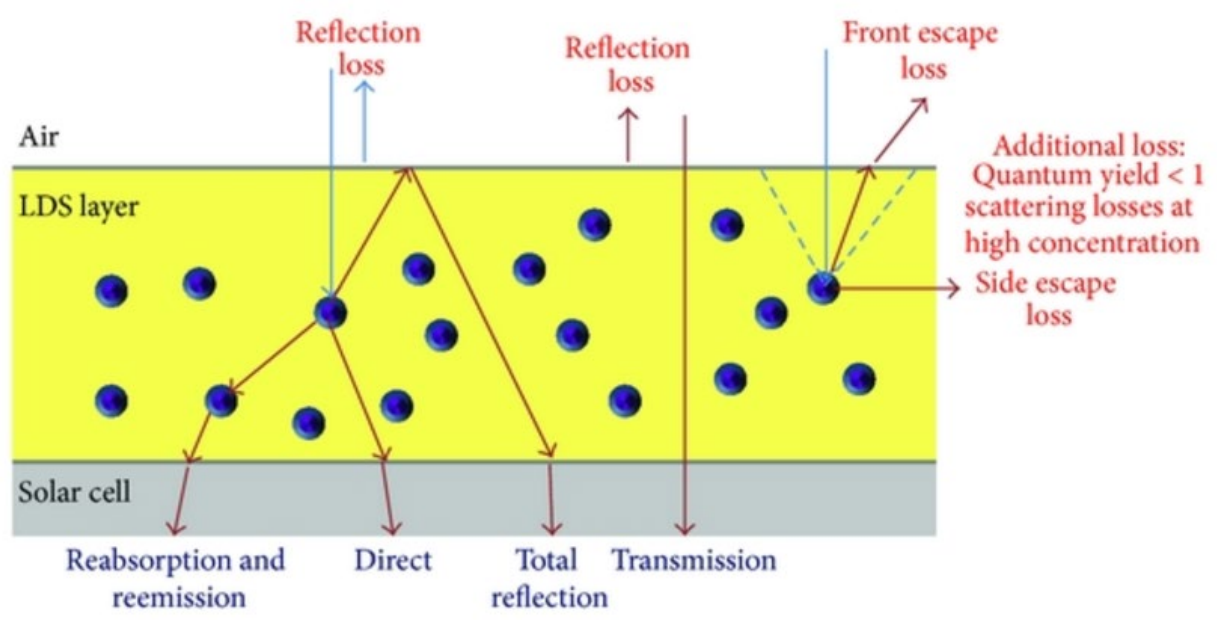

Figure 1. Schematic diagram of solar cell $\&$ attached LDS layer with possible optical paths for incident light [2]

Incident solar radiation can be absorbed and re-emitted back to the solar cell, where it is then either directly transmitted to the cell or undergoes internal reflection, at the LDS/Air boundary before reaching the cell. Light can be re-absorbed and re-emitted by other luminescent particles before reaching the PV cell, or lost entirely through the escape cone and from the side of the layer. The introduction of an LDS layer does introduce additional loss mechanisms which include; parasitic absorption from the host material, loss through reflection and scattering losses from luminescent material at higher concentrations [5]. An ideal LDS layer would contain a luminescent species where emission and absorption regions would not overlap so as to offset re-absorption. It would also have a quantum yield of 1 , meaning for every incident photon absorbed one is also emitted [6].

A type of luminescent material which can be utilised in the fabrication of LDS layers is an organic dye such as the Lumogen Violet 570, the focus of this study. Organic dyes are a suitable choice for many reasons. They exhibit high absorption coefficients, close to unity luminescent quantum efficiency, and are easily dissolved in polymers [4].

\section{Materials and Methods}

Materials: Host Materials (PVB \& PMMA). The host material should ideally have a very high transmittance and a low scattering in the spectral area of interest. This is the $300-500 \mathrm{~nm}$ range for the Silicon Solar Cell used in this study. The host materials studied in this paper are Polyvinyl Butyral (PVB) and Poly-Methyl Methacrylate (PMMA). They were chosen for their solubility and their optical characteristics. Figure 2, shows the transmission of the polymers measured using LabSphere Integrated system.

As it can be observed from Fig. 2, both polymers have transmission above $92 \%$ which make them suitable candidates for LDS layer application. 


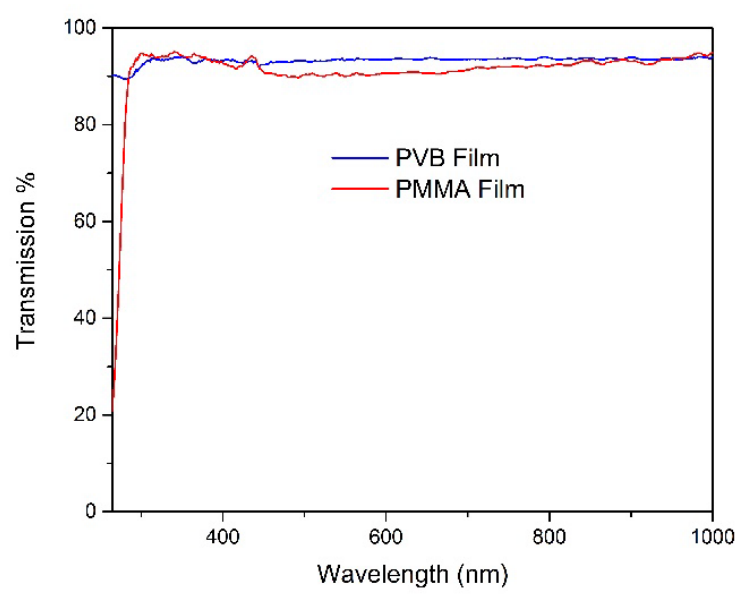

Figure 2. PVB \& PMMA Transmission spectra for 10 micron layer

Materials: Organic Dyes. Lumogen Violet 570 was selected for this study as Violet 570 has a high absorption coefficient, is relatively easy to process with polymers, and was found to have high luminescent quantum yield of up to 99\% [3]. These dyes have also had their photostability increased over recent years meaning that they can remain stable over many years of exposure to UV light [5].

Method: Host Material Preparation. Both PVB and PMMA were dissolved into chloroform to produce the initial polymer. The ratios were different however as the PVB - Chloroform mix was a 1 to 10 ratio (1g PVB for every 10ml Chloroform) whereas the PMMA - Chloroform was a 2.3 to 10 ratio solution. The PVB solution was stirred at $50^{\circ} \mathrm{C}$ at $700 \mathrm{rpm}$ for approximately 40 minutes whilst the PMMA solution was stirred at room temperature at $500 \mathrm{rpm}$ for between $40-60$ minutes. These solutions were then filtered so that replicable samples could be produced.

After the stock solutions had been filtered, the lumogen dyes were included directly to the samples after being measured in percent weight quantities. Different weight percentages ranging from 0.01 to $0.5 \mathrm{wt} \%$ were tested.

Method: LDS Layer Preparation. A spin-coating (G3P-12 Systems) technique was used for the fabrication of LDS layers. Mono-crystalline silicon cells $(2 \times 2 \mathrm{~cm}$ Solar Capture) were used for assessing the downshifting effect of the LDS layers. $10 \mu \mathrm{m}$ and $60 \mu \mathrm{m}$ thick films were directly spin-coated onto the top of the Si solar cells for $180 \mathrm{~s}$ at $1000 \mathrm{rpm}$ and $750 \mathrm{rpm}$ respectively. The thickness of these cells were measured at different points using a micrometer and the average was calculated.

Method: Absorption and Emission Measurements. The PerkinElmber Lambda 35 is a double beam UV/Vis spectrometer. It contains deuterium and halogen lamps pre-aligned with a wavelength range of 190 to $1100 \mathrm{~nm}$. The slit width has four options of $0.5 \mathrm{~nm}, 1 \mathrm{~nm}, 2 \mathrm{~nm}$, and $4 \mathrm{~nm}$. The software automatically switches the lamps at $326 \mathrm{~nm}$. The absorbance wavelength accuracy is $\pm 0.1 \mathrm{~nm}$ [7]. this machine was used to measure the absorption characteristics of the organic dye in the down-shifting layers. The Horiba FluroMax-4 is a spectrofluorometer used to measure the emission wavelengths in this study. It contains a xenon arc lamp, which passes through a monochromator. The monochromator selects a specific wavelength band as set by the user for the sample as the excitation wavelength. This spectrum is directed onto the sample, of which the luminescence from the sample is then directed to a second monochromator for emission, which selects a band of wavelengths and shines this onto the detector for the user to view the data.

Method: EQE Measurements. The EQE of a solar cell is the ratio of the number of charge carriers that are collected by the solar cell to the number of photons of a given wavelength entering into the solar cell [8]. The EQE of SpeQuest Quantum Efficiency has been used in this study. The setup is a combination of a xenon light source with a range of 300 to $1100 \mathrm{~nm}$, an IV converter, a bias light driver, a monochromator, and a chopper. The diameter of the light spot is from $2 \mathrm{~mm}$ up 
to $4 \mathrm{~mm}$, directed at the centre of the cell, measuring the photocurrent generated by the cell at $10 \mathrm{~nm}$ steps in wavelength. A calibrated silicon detector was used to calibrate for the measurements of spectral responsivity by determining the power in the probe beam as a function of wavelength. The system is entirely automated through a computer-based interface using the Photor software program.

\section{Results \& Discussion}

Figures 3 and 4 show the absorption and emission spectra of different concentrations of Violet/PVB LDS layers, while these spectra for the Violet/PMMA LDS layers are presented in Fig 5 and 6.

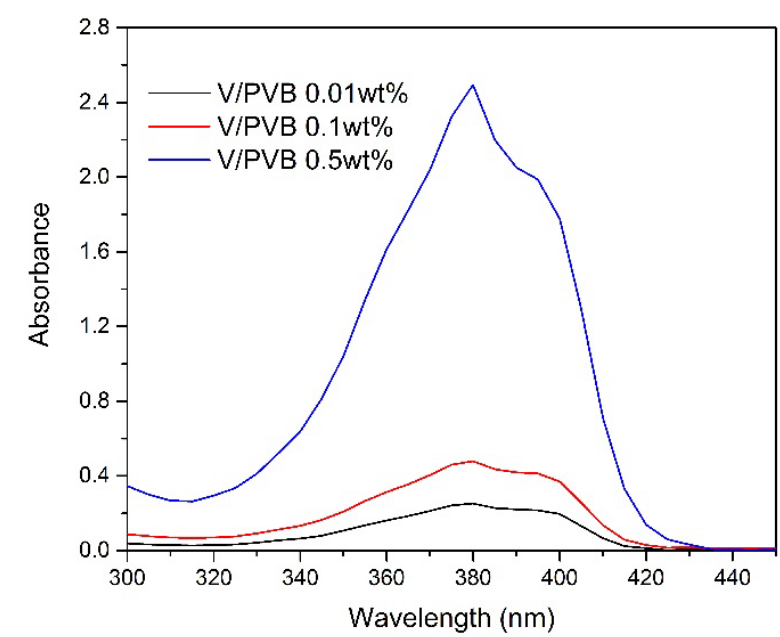

Figure 3. PVB and Chloroform Absorption Spectra for Si Solar Cell with Varying Concentration

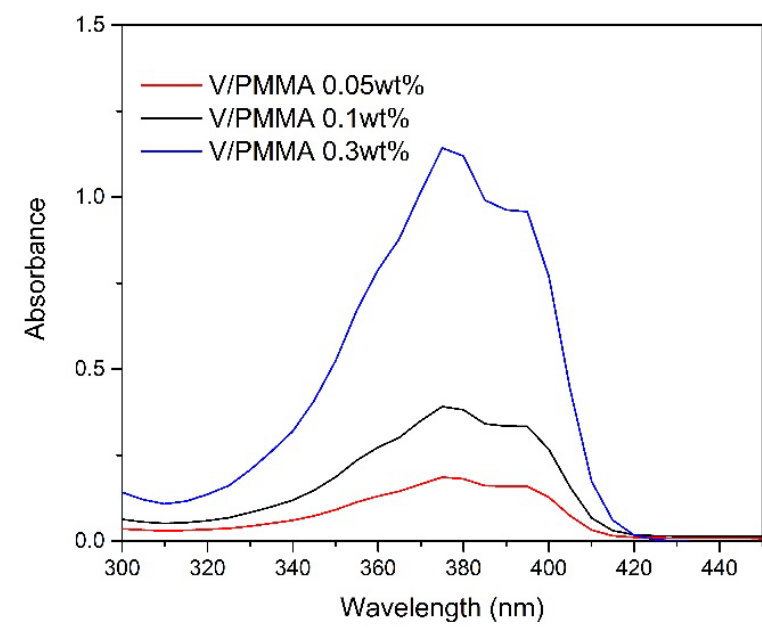

Figure 5. PMMA and Chloroform Absorption Spectra for Si Solar Cell with Varying Concentrations

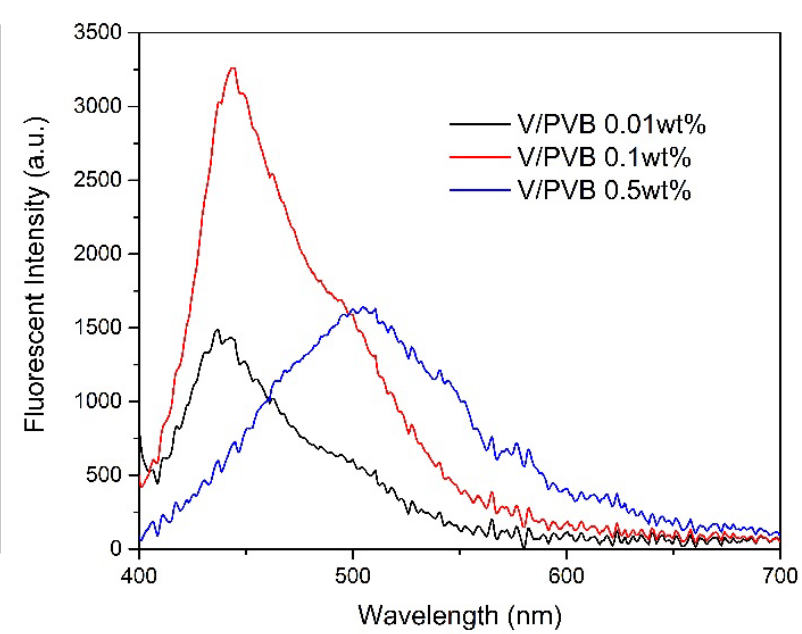

Figure 4. PVB and Chloroform Emission Graph for Si Solar Cell with Varying Concentrations

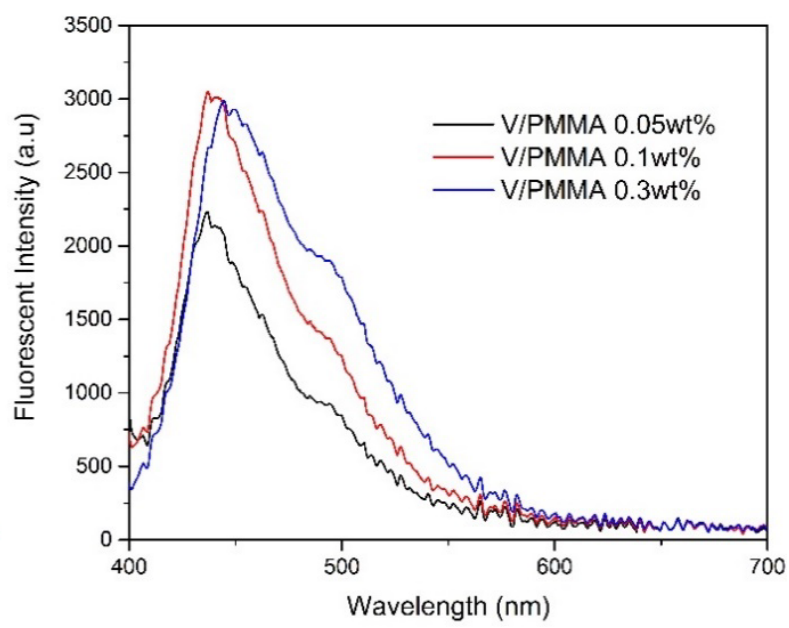

Figure 6. PMMA and Chloroform Emission Spectra for Si Solar Cell with Varying Concentrations

The results presented in Fig. 3 and Fig. 5, show that when the concentration of dye is increased, the absorption increases accordingly. This corresponds with a rise in the emission peaks, as shown in figures $4 \& 6$. However, this effect only occurs up to a certain point, after which the emission peak begins to shift to the larger wavelength region and gradually diminish. An example of which can be seen for PVB's emission spectrum in figure 4. Here, for concentrations from $0.01 \mathrm{wt} \%$ to 
$0.1 \%$ there is a peak emission in the 430-450nm range, although, when the concentration is increased as far as $0.5 \mathrm{wt} \%$ it can be seen that the peak has diminished, is red shifted and is now emitting in the 500-520nm range. It can be observed that the emission peak has dropped from its max value of $3.26 \times 10^{3}$ a.u. at $0.1 \mathrm{wt} \%$, to $1.63 \times 10^{3} \mathrm{a}$.u. at $0.5 \mathrm{wt} \%$. This could be due to the reabsorption of the luminescent species as a result of the high concentration used [9]. The results show that the current optimum concentration for PVB \& PMMA is $0.1 \mathrm{wt} \%$. It is expected that with further testing the optimal concentration for both polymers will be further improved.

As can be seen in figures $3 \& 5$ Violet's absorption range is between $320-420 \mathrm{~nm}$, which makes it suitable for LDS layers. The emission range of Violet is between 400-550nm when the layers are excited at a wavelength of $380 \mathrm{~nm}$. The peak absorption was $380 \mathrm{~nm}$ for both polymers and the peak emission was measured to be $443 \mathrm{~nm}$ for PVB and 436nm for PMMA giving a Stokes shift of $63 \mathrm{~nm}$ $\& 56 \mathrm{~nm}$, respectively.

EQE measurements were performed on the optimised concentrations for both PMMA \& PVB. Measurements were taken for a bare Si solar cell and the Si solar cells with LDS layers coated on top. Figures $7 \& 8$ shows the full range of measurements from $300-1100 \mathrm{~nm}$.

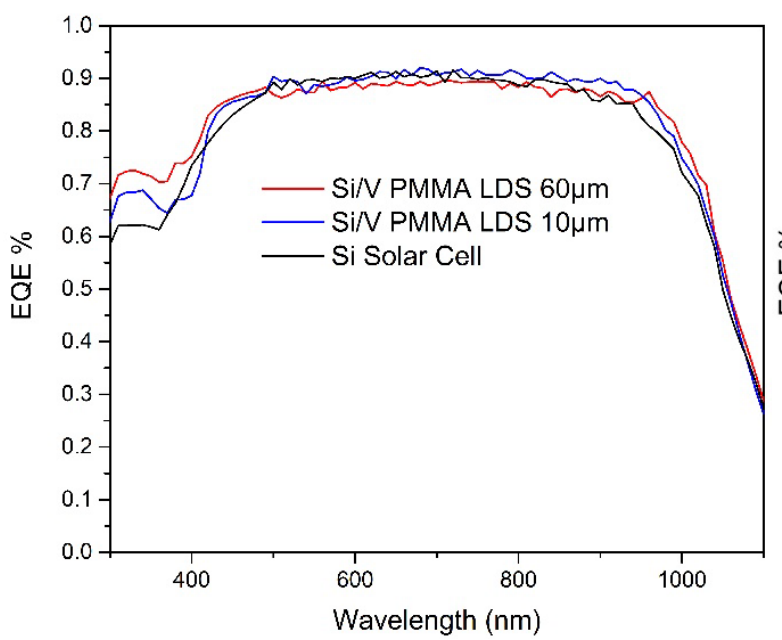

Figure 7. PVB \& Chloroform EQE for Si Solar Cell with Varying Thickness

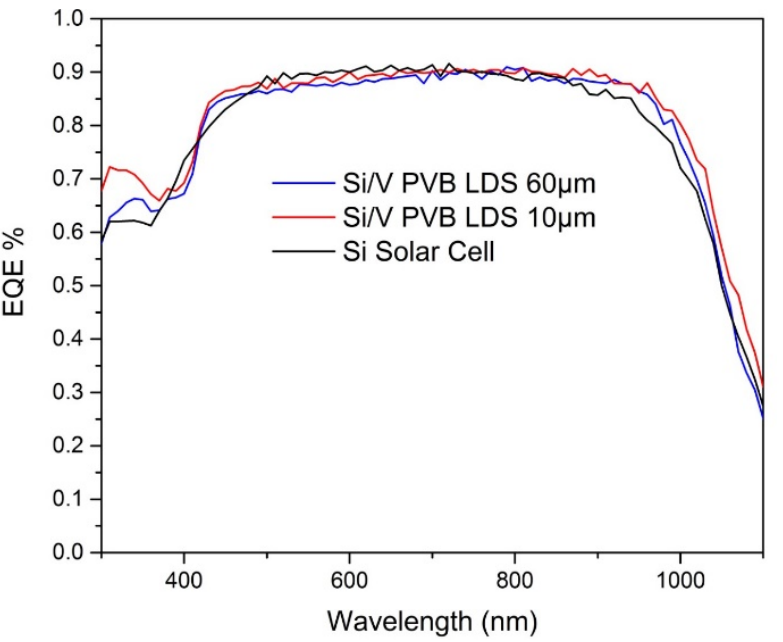

Figure 8. PMMA \& Chloroform EQE for $\mathrm{Si}$ Solar Cell with Varying Thickness

Atter calculatıng the ettıciency of the Si solar cells from the EQE graphs above, it was discovered that the best overall improvement in efficiency was in the $10 \mu \mathrm{m}$ PVB layer with a $+2.9 \%$ efficiency. This was followed by the $10 \mu \mathrm{m}$ PMMA layer with an increase of $+2.1 \%$. The thicker $60 \mu \mathrm{m}$ layers gave efficiency increases of $+1.5 \%$ and $+1.8 \%$ respectively.

\section{Conclusions}

Absorption and emission spectra measurements of Lumogen Violet 570 have shown that the dye is suitable for an LDS layer application. The optimum concentration was found to be $0.1 \mathrm{wt} \%$ for the range of LDS layers investigated in this paper. The future work will involve study of a wider range of concentrations in order to improve the optimal efficiency. External Quantum Efficiency (EQE) measurements have shown that an increase in the short wavelength response of the silicon solar cell was achieved due to the presence of Lumogen Violet dye. It has been found in the EQE results that the thinner layers $(10 \mu \mathrm{m}$ thickness) perform better compared to the thicker layers $(60 \mu \mathrm{m}$ thickness). The EQE enhancement for the target wavelength range, between 300 to $420 \mathrm{~nm}$, was calculated due to this being the range in which the silicon solar cell had the poorest spectral response. It was found that for thin V/PVB LDS layer the EQE came to 5.2\% while for V/PMMA, it was $10.5 \%$.

This study will be continued in order to characterise more efficient LDS layers with various dye concentration ratio in the larger scale. After the large scale cells have had their concentration 
optimised, metal nanoparticles will be optimised and added to the layers. This should further increase cell efficiency through plasmonic interaction.

\section{Acknowledgements}

The authors would like to acknowledge the funding from the European Research Council grant entitled PEDAL: Plasmonic enhancement of advanced luminescent solar devices (13379: 203889), funding from Science Foundation Ireland (SFI).

\section{References}

[1] M. Ram, et al. 2019. Introduction in Report Global Energy System Based On 100\% Renewable Energy, page 1.

[2] H. Ahmed: External Quantum Efficiency Improvement with Luminescent Downshifting Layers: Experimental and Modelling, School of Engineering, Trinity College Dublin, Dublin, Ireland. 2016.

[3] A. Ahmed, et al.: Advances in Energy Research, Vol. 1 (2013) p. 117.

[4] E. Klampaftis, et al.: Solar Energy Materials \& Solar Cells, Vol.93 (2009) p. 1182.

[5] H. Ahmed: Thesis. Materials Characterization and Plasmonic Interaction in Enhanced Luminescent Down-Shifting Layers for Photovoltaic Devices, School of Physics, College of Science and Health, Dublin Institute of Technology. Ireland. 2014.

[6] S. Gilligan: Enhancing Solar Cell Efficiency by Using Luminescent Down-Shifting Layers, School of Physics, College of Science and Health, Dublin Institute of Technology. 2016.

[7] PerkinElmerLtd (2000). PerkinElmer Lambda 35 Manual.

[8] C. Hu, and R.M. White: Solar Cells: From Basic to Advanced Systems. New York, McGrawHill, USA1983.

[9] S. Gilligan: Material Characterisation of Luminescent Down-Shifting Layers for Solar Cells Application. Department of Civil, Structural and Environmental Engineering, Trinity College Dublin. 2018. 
Reproduced with permission of copyright owner. Further reproduction prohibited without permission. 